(1)

CrossMark

\title{
The Global Lung Function Initiative (GLI) Network ERS Clinical Research Collaboration: how international collaboration can shape clinical practice
}

\author{
Graham L. Hall ${ }^{1,2}$ and Sanja Stanojevic ${ }^{3,4}$ on behalf of the GLI Network \\ Executive $^{5}$
}

Affiliations: ${ }^{1}$ Children's Lung Health, Telethon Kids Institute, Perth, Australia. ${ }^{2}$ School of Physiotherapy and Exercise Science, Curtin University, Perth, Australia. ${ }^{3}$ Translational Medicine, Hospital for Sick Children, Toronto, ON, Canada. ${ }^{~}$ Institute of Health Policy, Management and Evaluation, University of Toronto, Toronto, ON, Canada. ${ }^{5} \mathrm{~A}$ full list of the GLI Network Executive can be found at the end of this article.

Correspondence: Graham L. Hall, Children's Lung Health, Telethon Kids Institute, P.0. Box 855, West Perth, WA, 6872, Australia. E-mail: graham.hallatelethonkids.org.au

@ERSpublications

The Global Lung Function Initiative is working to improve the interpretation of lung function: an update on current work and the opportunities for further engagement http://ow.ly/kCXu30mYyAK

Cite this article as: Hall GL, Stanojevic S. The Global Lung Function Initiative (GLI) Network ERS Clinical Research Collaboration: how international collaboration can shape clinical practice. Eur Respir J 2019; 53: 1802277 [https://doi.org/10.1183/13993003.02277-2018].

\section{Rationale}

The diagnosis and management of lung disease includes assessments of lung function. However, using lung function data accurately mandates appropriate and meaningful interpretation of the outcomes that are personalised to the individual patient. For decades it was common for the same lung function result to be interpreted differently depending on the laboratory where the test was done, with wide discrepancies in what was defined as the normal range of values observed in a healthy population [1-3]. With more than 400 published reference equations characterising how lung function changes with size and age in healthy people, pulmonary function professionals were left with a difficult decision: which reference equation is most appropriate for my population? This lack of standardisation contributes to incorrect diagnosis, with significant economic, health and quality of life consequences. The Global Lung Function Initiative (GLI) Network was established to address this very issue, and provides a robust and standardised approach to improve the way in which lung function tests are interpreted around the world $[4,5]$. The Network evolved as a result of international collaboration and altruism between researchers, clinicians and industry partners.

\section{The GLI Network: an ERS Clinical Research Collaboration}

The GLI Network was formally established as a European Respiratory Society (ERS) Clinical Research Collaboration (CRC) [6] in 2016, to build upon previous ERS sponsored task force projects (table 1). The GLI Network sits within the airway disease theme of the ERS Research Agency and directly spans nearly all aspects of clinical research and global respiratory medicine. The GLI Network has become the largest resource for reference values for routine lung function testing ever assembled. Currently, the GLI 2012 reference equations for spirometry [7] and GLI 2017 reference equations for transfer factor of the lung for carbon monoxide (TLCO) [8] are endorsed by all major respiratory societies and recommended by several 


\begin{tabular}{lcc}
$\begin{array}{l}\text { TABLE } 1 \text { Summary of Global Lung Function Initiative (GLI) Network projects } \\
\text { Project }\end{array}$ & Start year & Status \\
\hline Spirometry & 2008 & Published [7] \\
Transfer factor for carbon monoxide & 2013 & Published [8] \\
Static lung volumes & 2016 & Publication in preparation \\
Exhaled nitric oxide & 2018 & Project initiated \\
Forced oscillation technique & 2018 & Project initiated
\end{tabular}

regional respiratory societies. In the short time since publication, the GLI equations have quickly become the gold standard for the interpretation of lung function outcomes.

As a result of the ERS CRC, the GLI Network has successfully grown its global network of collaborators and established a permanent data repository, maintained by the ERS. The GLI data capture platform securely stores lung function data from healthy individuals for multiple lung function tests, and can be easily amended to include additional tests. This will allow for routine updates of the equations to include additional groups and contemporary data. Approved governance, data sharing and data use agreements facilitate the collection and sharing of this rich data resource with researchers and the wider respiratory community. Through partnership with the European Lung Foundation (ELF), the GLI Network continues to improve resources for patients, educators and clinicians, including multi-language patient information leaflets and videos (for example see [9]). Importantly, the GLI CRC has facilitated the development of dissemination tools (including a web-based and mobile app calculator) and broader implementation of the GLI equations, which make it possible for a greater number of respiratory healthcare professionals to better interpret lung function test results. The GLI Network has grown to over 400 members, including data contributors, manufacturers of pulmonary function equipment and those interested in improving how lung function is interpreted. Each year, a GLI Network workshop is held at the ERS International Congress to bring together members and discuss challenges and areas of priority for the Executive to focus on. Importantly, everyone involved in the GLI Network contributes time and effort on an entirely voluntary basis.

The GLI Network will continue to work towards its aim of improving how lung function tests are interpreted by collating existing data from healthy individuals to produce robust reference equations across the lifespan. New initiatives include all-age reference equations for static lung volumes, exhaled nitric oxide and forced oscillation technique (table 1). Beyond providing standardised reference equations, the GLI Network has reignited debate and discussion around how lung function is interpreted, particularly with respect to using an age-specific lower limit of normal to differentiate between health and disease accurately across the entire lifespan. Visual representations of lung function test results have helped to raise awareness of the uncertainty of results, and to interpret results in the context of the range of values that are observed in health (figure 1). A complete summary of the history of the GLI Network and practical solutions to integrating available reference equations into clinical practice were previously summarised in the ERS educational publication, Breathe $[4,5]$.

\section{The GLI Network welcomes new members and contributors}

The GLI Network is not without its challenges. Despite its name, GLI spirometry lacks data from certain populations, namely those on the African continent, South Asia and India, and Latin America. The TLCO equations are only available for those of White European descent. Several efforts have been made to collect and summarise data in the under-represented populations [10-12]; while these studies provide critical information regarding the generalisability of GLI equations in these populations, the conclusions have consistently been that prospective, high-quality data are needed. Through our partnership with ELF, the GLI Network is working to integrate the Healthy Lungs for Life programme (www.healthylungsforlife.org) and the ERS Spirometry Training Programme (www.ersnet.org/professional-development/spirometrytraining-programme), to broaden the scope and impact of all three of these programmes, and to facilitate the collection of high-quality normative data around the world. This new collaborative platform will be essential for current and new GLI members to collect and collate data from under-represented populations to derive more appropriate reference equations for these regions and ethnicities. If you are interested in hosting a Healthy Lungs for Life event in your community, and contributing data to the GLI database, please contact the ELF or GLI teams for more details.

For the GLI Network to continue to succeed and improve the interpretation of lung function, it is vital that our network members continue to work together to further improve how lung function is reported 


\section{Results}

These results are re-calculated automatically

when you update the patient information.

$\mathrm{FEV}_{1}$

\begin{tabular}{|l|l|}
\hline Predicted & 3.72 \\
\hline LLN & 2.98 \\
\hline Z score & 1.10 \\
\hline \% predicted & $113 \%$ \\
\hline
\end{tabular}

FVC

\begin{tabular}{|l|l|}
\hline Predicted & 4.47 \\
\hline LLN & 3.58 \\
\hline Z score & $\mathbf{0 . 7 6}$ \\
\hline \% predicted & $\mathbf{1 1 0 \%}$ \\
\hline
\end{tabular}

\section{$\mathrm{FEV}_{1} / \mathrm{FVC}$}

\begin{tabular}{|l|l|}
\hline Predicted & 0.84 \\
\hline LLN & 0.72 \\
\hline Z score & 0.32 \\
\hline \% predicted & $102 \%$ \\
\hline
\end{tabular}

FIGURE 1 The Global Lung Function Initiative (GLI) mobile app and visualisation tool lavailable from www.lungfunction.orgl permits the user to calculate the lower limit of normal (LLN), Z score and predicted values for forced expiratory volume in $1 \mathrm{~s}$ (FEV 1 ), forced vital capacity (FVC) and $\mathrm{FEV}_{1} / \mathrm{FVC}$. 
and interpreted across all age groups and ethnicities around the world. Further information about the GLI Network can be found at www.lungfunction.org. The GLI Network welcomes members from all over the world. Data collection is open for all lung function tests. If you have high-quality pulmonary function data from healthy individuals and are eager to join the GLI Network, please visit the website for further details.

Acknowledgements: The GLI Network wishes to thank the 400 GLI Network members who have generously transferred their lung function data, without which none of our achievements would have been possible. Finally, we pay our lasting respect to Philip Quanjer for his drive and initiative.

Members of the GLI Network Executive: Janet Stocks, Brendan Cooper, Irene Steenbruggen, John Henderson, Carlos A. Vaz Fragoso, Gail McAvay, Laura Gochicoa Rangel, Refiloe Masekela, Le Thi Tuyet Lan, Pippa Powell and Meredith McCormack.

Conflict of interest: G.L. Hall reports grants from the European Respiratory Society to fund the activities of the GLI Network as a Clinical Research Collaboration. S. Stanojevic reports grants from the European Respiratory Society to fund the activities of the GLI Network as a Clinical Research Collaboration.

Support statement: Funding was received from the European Respiratory Society. Funding information for this article has been deposited with the Crossref Funder Registry.

\section{References}

1 Rosenfeld M, Pepe MS, Longton G, et al. Effect of choice of reference equation on analysis of pulmonary function in cystic fibrosis patients. Pediatr Pulmonol 2001; 31: 227-237.

2 Quanjer PH, Borsboom GJ, Kivastik J, et al. Cross-sectional and longitudinal spirometry in children and adolescents: interpretative strategies. Am J Respir Crit Care Med 2008; 178: 1262-1270.

3 Quanjer PH, Weiner DJ. Interpretative consequences of adopting the Global Lungs 2012 reference equations for spirometry for children and adolescents. Pediatr Pulmonol 2014; 49: 118-125.

4 Cooper BG, Stocks J, Hall GL, et al. The Global Lung Function Initiative (GLI) Network: bringing the world's respiratory reference values together. Breathe 2017; 13: e56-e64.

5 Stanojevic S, Quanjer P, Miller MR, et al. The Global Lung Function Initiative: dispelling some myths of lung function test interpretation. Breathe 2013; 9: 462-474.

6 Brightling C, Genton C, Bill W, et al. ERS Clinical Research Collaborations: underpinning research excellence. Eur Respir J 2018; 52: 1801534.

7 Quanjer PH, Stanojevic S, Cole TJ, et al. Multi-ethnic reference values for spirometry for the 3-95-yr age range: the global lung function 2012 equations. Eur Respir J 2012; 40: 1324-1343.

8 Stanojevic S, Graham BL, Cooper BG, et al. Official ERS technical standards: Global Lung Function Initiative reference values for the carbon monoxide transfer factor for Caucasians. Eur Respir J 2017; 50: 1700010.

9 European Lung Foundation. Testing your lungs: spirometry. www.europeanlung.org/assets/files/en/publications/ spirometry-en.pdf Date last updated: July 2018.

10 Arigliani M, Canciani MC, Mottini G, et al. Evaluation of the Global Lung Initiative 2012 reference values for spirometry in African children. Am J Respir Crit Care Med 2017; 195: 229-236.

11 Lum S, Bountziouka V, Quanjer P, et al. Challenges in collating spirometry reference data for South-Asian children: an observational study. PLoS One 2016; 11: e0154336.

12 Quanjer PH, Kubota $\mathrm{M}$, Kobayashi $\mathrm{H}$, et al. Secular changes in relative leg length confound height-based spirometric reference values. Chest 2015; 147: 792-797. 